\title{
IR EXCESSES OF LATE-TYPE WC STARS: NO DUST NEEDED IN MOST CASES
}

\author{
T. NUGIS \\ Tartu Astrophysical Observatory, EE2444 Tõravere, Estonia
}

\begin{abstract}
Many late-type WC stars exhibit strong IR excesses which have usually been explained by hot dust radiation. Alternatively, we found that free-free and free-bound radiation of a small number of high density clumps beyond the effective radio-emission zone may give rise to "dust-like" IR excesses.
\end{abstract}

Key words: stars: Wolf-Rayet - WCL - IR excesses - clumped shells

About $50 \%$ of WC8 stars, $85 \%$ of WC9 stars, the only galactic WC10 star and episodically some binary WC7 stars show IR excesses which have usually been interpreted as dust radiation (Williams et al. 1987). Modelling studies of dust shells have indicated that dust must originate in the wind regions situated near the effective radio-emission formation zone, i.e., in the region where electron temperatures are of the order of $7000-10000 \mathrm{~K}$ and the matter is predominantly ionized. Dust formation is rather complicated in such a hostile environment (stellar temperatures of WC9 stars are in the range $25000 \mathrm{~K}-30000 \mathrm{~K}$ and of WC8 stars around $40000 \mathrm{~K}$ ). The purpose of the present communication is to look for other possibilities than dust to explain the observed IR excesses in WCL stars.

Some peculiar structure of the winds (deceleration zones, coronal zones, small-scale density inhomogeneities) can make the spectral energy curves in the IR range smoother. Yet it is impossible to achieve $f_{\nu}\left(\lambda_{2}\right)>f_{\nu}\left(\lambda_{1}\right)$ with $\lambda_{2}>\lambda_{1}$. Let us look for the possibility that the observed IR excesses are due to additional radiation from the moving shell lying somewhere beyond the effective radio-emission zone of WCL stars. Let the shell consist of a small number of high density clumps and of a low density gas. Both these shell components must not contribute to the radio fluxes and to line emission because radio-fluxes and line fluxes of WCL stars with and without IR excesses are of the same relative strength. If the density of clumps is high enough, then they are optically thick for $f-f$ and $b-f$ radiation at radio wavelengths and may remain optically thick even at IR wavelengths. The source function of shell radiation increases much more steeply with the increase of frequency than the radiation power of the wind and therefore the clumps may contribute to the IR continua. Continuum fluxes of WCL stars at IR and radio wavelengths are due to wind and moving shell contributions (only radiation from high density clumps of the shell is important). The contribution of the wind to the total IR flux of WCL stars showing IR excesses was 
found by assuming that for them the relative contribution to the total flux at different wavelengths is the same as for the WCL stars without IR excesses. The matter in the clumps is only partially ionized. In such conditions the electron temperature is lower than in the ionized wind region where the radio-fluxes are effectively formed (compare the temperatures of galactic $\mathrm{H} \mathrm{I}$ and $\mathrm{H}$ II regions). In the IR spectral range the opacity of clumps is dominated by $b-f$ transitions at low electron temperatures. Assuming that the high density clumps, which are optically thick at radio wavelengths, remain optically thick also in the IR spectral region and that the populations of the levels contributing to $b-f$ radiation are not strongly different from the LTE values, we can explain the IR excesses of WCL stars with sharp maxima at 3.6-4.8 $\mu \mathrm{m}$ (most of the WCL stars with IR excesses) by the radiation of clumps having electron temperatures around 1000-1500 K.

In the case of WCL stars with plateau-like excesses (the stars WR104, WR112, WR118), reasonable fits to the observed IR continua can be obtained by assuming that some clumps remain optically thick and some of them become optically thin in the IR spectral range. If there exist departures from LTE populations of levels contributing to the $b$ - $f$ radiation at IR wavelengths then the agreement with the observed fluxes can be obtained with higher electron temperatures. In the case of stars with sharp maxima again all the clumps must remain optically thick in the IR spectral range, but now jumps appear in the series edges. In the case of stars with plateau-like maxima the jumps cannot be strong because the absorption and emission jumps nearly cancel out. The contribution of clumps to the line emission ought to be negligible: $F_{\nu}^{l} / F_{\nu}^{c} \approx 2 \delta R_{s h} S_{l} / R_{s h} S_{c}$, where $\delta R_{s h}$ is the geometrical thickness of the shell which ought to be much smaller than $R_{s h}$. Low density matter of the moving shell may contribute to line absorption in some cases. The violet-shifted shell lines of Fe III observed in the spectrum of WC9 star WR103 (van der Hucht \& Conti 1981) seem to be the examples of such lines. No doubt that dust may contribute to IR fluxes of some WCL stars, and the high density clumps which are predominantly neutral, may be suitable places for dust formation.

Episodic formation of "dust-like" IR excesses in some WC7 binary systems may also be explained by the presence of high density clumps far from the stellar surface. The matter of these clumps must be neutral during most of the time and only at some epochs when additional strong radiation reaches them they may become partly ionized and give rise to IR excesses.

\section{References}

van der Hucht K.A., Conti P.S. 1981, in: C. Chiosi R. Stalio (eds.), Effects of Mass Loss on Stellar Evolution Proc. IAU Coll. 59 (Dordrecht: Reidel), p. 35

Williams, P.M., van der Hucht, K.A., Thé, P.S. 1987, $A \& A$ 182, 91 